Questions vives

\section{Questions Vives}

Recherches en éducation

$n^{\circ} 22 \mid 2014$

Questionner le curriculum en éducation physique et sportive : Quelle dynamique en contexte? Quelle autonomie?

\title{
L'implémentation des nouveaux programmes par compétences en EPS au Bénin : une analyse des contenus enseignés
}

The implementation of the competence based physical education new

curriculum in Benin: an analysis of the content really taught

Basile Agbodjogbé, Kossivi Attiklémé et Carlos Atoun

\section{(2) OpenEdition}

Journals

Édition électronique

URL : http://journals.openedition.org/questionsvives/1579

DOI : $10.4000 /$ questionsvives. 1579

ISSN : $1775-433 \mathrm{X}$

Éditeur

Université Aix-Marseille (AMU)

Édition imprimée

Date de publication : 15 décembre 2014

ISBN : 978-2-912643-46-9

ISSN : 1635-4079

\section{Référence électronique}

Basile Agbodjogbé, Kossivi Attiklémé et Carlos Atoun, «L'implémentation des nouveaux programmes par compétences en EPS au Bénin : une analyse des contenus enseignés », Questions Vives [En ligne], $n^{\circ} 22$ | 2014, mis en ligne le 16 février 2015, consulté le 30 avril 2019. URL : http://

journals.openedition.org/questionsvives/1579; DOI : 10.4000/questionsvives.1579

Ce document a été généré automatiquement le 30 avril 2019.

\section{cc) (†)}

Questions Vives est mis à disposition selon les termes de la licence Creative Commons Attribution -

Pas d'Utilisation Commerciale - Pas de Modification 4.0 International. 


\title{
L'implémentation des nouveaux programmes par compétences en EPS au Bénin : une analyse des contenus enseignés
}

\author{
The implementation of the competence based physical education new \\ curriculum in Benin: an analysis of the content really taught
}

Basile Agbodjogbé, Kossivi Attiklémé et Carlos Atoun

\section{Introduction}

Le vaste mouvement curriculaire engagé depuis plus de deux décennies en Afrique a permis à plusieurs Etats de réorienter leur système éducatif vers l'approche par les compétences. Le Bénin n'est pas resté en marge de cette révolution curriculaire » et s'est engagé dans une refonte de ces programmes dans toutes les disciplines. Les nouveaux programmes d'enseignement ainsi adoptés sont fondés sur l'approche par les compétences (APC). Ils ont été élaborés dans toutes les disciplines, à l'enseignement primaire comme au secondaire, par un groupe d'enseignants composé d'inspecteurs et de conseillers pédagogiques appuyés par moments par des experts internationaux. D'origine canadienne et belge, ces experts ont apporté leurs expertises dans l'élaboration du cadre général orientant la refondation curriculaire. Ce cadrage théorique fortement centré sur un modèle "importé » n'a pas réellement pris en considération toutes les pesanteurs socioculturelles et structurelles des pays africains en général et celles du Bénin en particulier. Ces pesanteurs influencent la mise en place des réformes curriculaires. Or, selon Crahay, Audigier et Dolz (2006), il est nécessaire de prendre en compte d'une part l'impact des traditions culturelles, des croyances, des convictions, des valeurs des enseignants, des décideurs politiques et des parents; ainsi que, d'autre part, celui du jeu des lobbys économique ou politique et des différentes structures centralisées (ou non) qui pèsent sur le développement curriculaire. La prise en compte de ces particularités 
sociétales sur le processus d'élaboration et d'implémentation du curriculum repose sur une double influence : celle des concepteurs et celle des facteurs contextuels, notamment relatifs aux praticiens.

2 Si la question des rapports aux savoirs des concepteurs (Attiklèmè, 2009) ainsi que le point de vue des acteurs de terrain (Agbodjogbé, Amade-Escot \& Attiklèmè, 2013) ont été déjà étudiés, nous nous intéressons dans cette étude à la façon dont un enseignant d'éducation physique et sportive (EPS), conseiller-pédagogique activement impliqué dans la réforme et à ce titre membre de la noosphère, implémente les contenus des nouveaux programmes en éducation physique et sportive (NPE-EPS) dans ses classes. Nous nous attachons particulièrement aux diverses transformations des contenus d'enseignement opérées par ce conseiller-pédagogique, aux influences des contrats institutionnels et didactiques lors de la gestion des transactions didactiques et de la co-construction des savoirs. Pour contextualiser le propos, il nous semble indispensable de caractériser le contexte d'enseignement béninois qui est passé en presque deux décennies d'un paradigme de transmission de connaissance selon le modèle de la pédagogie par objectif (PPO) à un paradigme dit de « la construction des savoirs » dans lequel l'élève est censé être placé au centre du processus selon les programmes par compétences.

\section{Contexte de l'étude}

3 Le Bénin a connu depuis les années 60 une série de réformes successives de son système éducatif. La réforme éducative dite du « programme national de l'édification de l'école nouvelle» (1972-1990) sous l'impulsion du mouvement révolutionnaire d'obédience marxiste a montré la nécessité d'installer un système d'éducation démocratique et patriotique pour l'enseignement d'une science et d'une technique modernes au service des intérêts du peuple. Dans cette perspective, le gouvernement militaire révolutionnaire d'alors a adopté le 23 juin 1975 l'ordonnance n 75-30 qui stipule que » l'école nouvelle doit être libérée de toute domination étrangère et de toute aliénation culturelle ; elle doit être intégrée au milieu social national, tout en constituant un centre promoteur de développement politique, économique, social et un moyen de statut collectif qui favorise la participation de tous à la production: elle doit être démocratique et populaire, obligatoire et gratuite, publique et laïque. Elle doit utiliser toutes les compétences intellectuelles et techniques disponibles dans la nation, et les structures traditionnelles d'enseignement pour associer la liaison de tous les ordres d'enseignement avec la vie pratique et le milieu social par l'initiation à la production ».

\subsection{De la pédagogie par objectifs à l'approche par les compétences : nécessité d'un changement de paradigme}

Dans la droite ligne de l'application de cette ordonnance, les programmes conçus mettent un accent particulier sur l'introduction progressive des langues maternelles dans l'enseignement. Elles sont considérées comme des matières d'enseignement et de véhicule des savoirs. L'enseignement doit faire une place de choix à l'EPS fondée sur la pratique effective du sport et de l'athlétisme en vue de donner à la nation des hommes équilibrés physiquement et intellectuellement. Dans toutes les disciplines les programmes formalisés définissent clairement les objectifs à atteindre, les activités d'enseignement et d'apprentissage et les tâches à exécuter selon les principes d'une 
pédagogie par objectifs (PPO). Malgré les résultats positifs de ce programme, des dérives sérieuses sont observées, tributaires des présupposés sous-jacents à cette approche tels que la recherche de comportements observables dans toutes les activités, un découpage très élémentarisé des contenus, le saucissonnage des unités d'apprentissage en de multiples objectifs opérationnels, l'émiettement des actions mentales, l'insuffisance de finalisation et de contextualisation des apprentissages scolaires (Agbodjogbé, 2013).

En EPS, les buts, les objectifs et les finalités sont déterminés par des intentions éducatives en tenant compte du niveau de l'élève. Les activités sont essentiellement choisies en fonction des possibilités qu'elles offrent dans leurs diversités, pour répondre à l'ensemble des finalités énumérées dans le domaine de la résistance physique, de la disposition motrice et de l'éducation globale. Après plusieurs années d'application de ce programme, et à l'issue du séminaire « Bilan de l'école nouvelle » en 1981, une inflexion est proposée : les programmes par objectifs sont abandonnés au profit de programmes par contenus. Dans la gamme étendue des activités sportives, ces derniers retiennent des activités telles que l'athlétisme en général, la gymnastique et les sports collectifs pratiqués tout le long de la scolarité. Ces activités physiques et sportives (APS) constituent la base même de l'enseignement de l'EPS, sans pour cela que les pratiques scolaires ne soient pour autant profondément transformées.

6 Les difficultés rencontrées, les insuffisances dénoncées imposent un changement de paradigme (Zomahoun, 2008) qui trouve ses sources dans la « Déclaration mondiale sur l'éducation pour tous " adoptée à Jomtien en 1990, opérationnalisée dans le «cadre d'action de Dakar» (UNESCO, 2000) à partir des indicateurs proposés par l'OCDE (2008). Pour dépasser la situation de sclérose éducative, accentuée par la crise économique mondiale des années 90, le Bénin s'engage alors avec l'appui de la Banque Mondiale sur une refonte de son système éducatif en adoptant à la suite de la Conférence des Forces Vives de la Nation (1990) les principes d'un curriculum basé sur le développement des compétences.

7 La mise en application des recommandations issues de cette conférence touche tous les ordres d'enseignement. Entreprise depuis 1993 au primaire et en 2000 au secondaire, cette réforme a pour ambition de former autrement les enfants béninois. Les fondements épistémologiques de la réforme stipulent que la compétence se développe dans et par l'action (Roegier, 2008; Jonnaert, 2002; Jonnaert, Ettayebi \& Defise, 2009). Il s'agit d'assurer la formation d'un citoyen de demain : « un citoyen autonome intellectuellement et physiquement équilibré, capable d'entreprendre, de se prendre en charge et d'apprendre tout au long de sa vie, un citoyen respectueux de la personne humaine, de la vie, de la vérité, et de la démocratie, animé d'un esprit de méthode, de coopération et du goût de la responsabilité, un citoyen gestionnaire de lui-même, gestionnaire de l'environnement et gestionnaire de la vie sociale » (Actes du forum sur le secteur de l'éducation, p. 4 ). La visée des nouveaux programmes par compétences consiste à rompre avec les formes d'enseignement magistral en usage, en donnant la priorité «aux compétences vues comme des savoir-agir, [à] une implication forte des apprenants, [à] un enseignant vu comme médiateur des savoirs » (Cros \& al., 2009, p. 7).

Cependant, la mise en œuvre de ces programmes conçus selon l'APC, n'est pas restée sans créer de difficultés aux acteurs de l'éducation (pour une évaluation critique de ces réformes curriculaires en Afrique, voir Cros et al., 2009). Au-delà de celles inhérentes à la clarification du concept de compétence, à la conception de l'apprentissage, à l'évaluation des apprentissages et aux fondements épistémologiques de l'approche, les facteurs 
contextuels ont aussi lourdement pesé dans la mise en œuvre des nouveaux programmes d'études. Par exemple au Bénin, la réforme ayant été mise en place dans des conditions organisationnelles et matérielles inchangées n'a pas été sans poser de problèmes aux enseignants d'EPS (Attiklèmè, 2009). Par ailleurs, l'explosion démographique galopante et certaines mesures d'accompagnement prises par les autorités béninoises (la gratuité de l'enseignement primaire, la réduction des frais de scolarité pour les filles) ont engendré un accroissement des effectifs des classes dans l'enseignement secondaire. La plupart des collèges béninois ont des effectifs pléthoriques et nombreuses sont les classes qui avoisinent 80 voire parfois 100 élèves. Du coup le ratio élèves /enseignant augmente avec pour corollaire une gestion difficile de la classe. Au vu de ces effectifs, les NP-EPS recommandent que les enseignants divisent le groupe-classe en deux : un groupe est en apprentissage sous la responsabilité de l'enseignant lors de la première moitié de séance (une heure et demie) pendant que l'autre est en "auto encadrement " sur un autre atelier. Une permutation à mi-séance est effectuée. Quelques travaux ont commencé à étudier les modalités d'implémentation de ces nouveaux programmes (Agbodjogbé, 2007, 2013 ; Ogueboule, 2008). Il ressort de leurs constats que la mise en œuvre des NPE-EPS reste difficile. Dans cet article nous rendons compte de la manière dont un enseignant d'EPS, particulièrement impliqué dans la réforme en tant que conseiller-pédagogique assurant son suivi, les met en œuvre dans une de ses classes. Nous précisons dans la section suivante quels sont les préconisations et les contenus proposés par les NPE EPS.

\subsection{Quelles acquisitions visent les NPE-EPS?}

9 Au-delà des grandes intentions éducatives, les NPE-EPS visent spécifiquement le développement des capacités, des habiletés issues de trois types de compétences (disciplinaire, transversale et transdisciplinaire) définies par les textes officiels communs à toutes les disciplines. Des connaissances et techniques en rapport avec les APS support de développement des compétences d'une part, et des valeurs d'ordre moral d'autre part, sont aussi énoncées dans ces textes. Dans le cadre de cet article qui porte sur les contenus enseignés en basket-ball par un enseignant conseiller pédagogique, le tableau ci-après présente les « attendus » des NPE-EPS en termes d'objets d'étude, d'acquisitions visées et de démarche d'enseignement et d'apprentissage.

Tableau 1 : Synthèse des « attendus » des NPE-EPS en basket-ball.

\begin{tabular}{|c|c|c|}
\hline Objet d'études & Acquisitions visées & Démarche \\
\hline \multirow{2}{*}{$\begin{array}{l}\text { Compétence } \\
\text { disciplinaire : } \\
\text { Pratiquer } \\
\text { collectivement des } \\
\text { activités } \\
\text { physiques : Basket- } \\
\text { ball. }\end{array}$} & $\begin{array}{l}\text { Les connaissances : Acquérir les } \\
\text { notions de règlement de l'APS, de } \\
\text { coopération, d'opposition, de } \\
\text { partenaire, d'adversaire, de système } \\
\text { de jeu, d'attaque, de défense. }\end{array}$ & $\begin{array}{l}\text { Résolution de problème : basée } \\
\text { sur la mise en œuvre des } \\
\text { capacités: Expression, analyse, } \\
\text { planification, exploration, prise } \\
\text { de position, exécution, } \\
\text { objectivation, amélioration, } \\
\text { réinvestissement. }\end{array}$ \\
\hline & $\begin{array}{l}\text { (passe et va, passe et suit) passe, } \\
\text { réception, pivot, feinte, dribble, } \\
\text { marquage, démarquage. }\end{array}$ & $\begin{array}{l}\text { Forme de travail: travail } \\
\text { individuel, travail de groupe, } \\
\text { travail collectif. }\end{array}$ \\
\hline
\end{tabular}




\section{Cadre théorique et problématique}

10 enseignant relève d'une analyse ascendante de la transposition didactique (SchubauerLeoni \& Leutenegger, 2005) qui stipule que les contenus mis à l'étude sont à rechercher dans les dispositifs d'étude proposés et dans l'activité effective du professeur et des élèves. Il s'agit de décrire comment le professeur met en scène le savoir et dirige l'étude des élèves in situ. L'analyse ascendante de la transposition didactique cherche donc à comprendre l'action conjointe des professeurs et des élèves relativement aux objets de savoir à enseigner et à apprendre (Amade-Escot, 2007). En ce sens notre recherche s'inscrit dans la cadre théorique de l'action conjointe professeurs-élèves, cadre qui modélise le fonctionnement ordinaire des systèmes didactiques (Sensevy, 2007). Les savoirs enseignés seront ici approchés dans le cadre de l'étude des gestes professionnels et des tâches professorales tels que définis par Chevallard (1997). En effet, cet auteur postule que la conscience didactique de l'enseignant s'actualise dans l'interaction avec les élèves autour de l'enjeu d'enseignement/apprentissage/évaluation. Ainsi se réfère-il à la notion d'assujettissement (Chevallard, 1991, 2002) qui met en évidence que les institutions traversées par les intervenants, influent sur leur rapport aux savoirs.

11 Ce cadrage théorique permet, selon nous, d'analyser comment est mise en œuvre la réforme curriculaire au Bénin et quelle est l'interprétation qui en est faite au niveau des pratiques. Les questions de recherches dont les suivantes : Comment sont mis en œuvre les contenus d'enseignement de la nouvelle matrice disciplinaire introduite par les NPEEPS ? A quelles pratiques scolaires de référence sont confrontés les élèves ? Quels sont les objets d'enseignement réellement étudiés en basket-ball en situation? Quelle épistémologie scolaire se dégage des pratiques observées?

\section{Méthodologie}

12 L'étude s'inscrit dans le protocole de Leutenegger (2009) qui stipule que l'objet de l'observation est le fonctionnement du système didactique et non les acteurs du système (enseignant ou élève). L'observation didactique s'intéresse ainsi aux comportements et aux interactions développées en contexte (Schubauer-Leoni \& Leutenegger, 2000) relativement à un morceau de vie de la classe, ici en lien avec la mise en place des NPEEPS.

Dans le cadre de cette étude, nous avons reconstruit sous forme d'une monographie les traits les plus saillants de l'action didactique de l'enseignant observé, dont nous rappelons qu'il a un statut de conseiller-pédagogique au sein du groupe de pilotage des NPE-EPS, mais qui a été observé en situation d'enseignement ordinaire avec une de ses classes. Nous nous sommes appuyés pour le faire sur des entretiens (ante et post) et les synopsis (Leutenegger, 2009) des trois séances de basket-ball observées. Ces entretiens ont permis de connaitre les intentions didactiques de l'enseignant, les objectifs choisis et leur atteinte. Quant aux observations, elles relèvent de ce que l'on peut appeler une observation de type ethnographique c'est-à-dire visant à décrire une culture particulière (ici un enseignant conseiller-pédagogique, membre de la noosphère en présence d'un objet d'enseignement : le basket-ball). 


\subsection{L'enseignant observé}

Il s'agit d'un enseignant conseiller-pédagogique âgé de 48 ans. Il totalise 22 ans d'expérience professionnelle. Membre de l'équipe d'encadrement pédagogique, il est concepteur des nouveaux programmes d'études (NPE) et acteur principal de leur implémentation. Il participe également à la confection des supports pédagogiques, notamment les planches de gymnastique et des cahiers d'activités. Ainsi, nous sommes en présence d'un sujet à double statut: un enseignant ordinaire capable de dérouler une séance de classe avec toutes les exigences prescrites par les instructions scolaires d'une part, et un enseignant ayant statut de conseiller pédagogique, porte-parole du législateur et garant du respect des normes institutionnelles, d'autre part. Il est donc possible que sa pratique enseignante soit par moment influencée par ses attributs de conseillerpédagogique, éléments centraux de son épistémologie pratique. Cet enseignant est par ailleurs, spécialiste de volley-ball, ce qui peut laisser penser qu'il dispose de solides connaissances dans l'enseignement des sports collectifs. Son expérience professionnelle (22 ans) pourrait aussi lui conférer une certaine expertise en matière d'enseignement de l'EPS.

\subsection{Les entretiens}

Deux types d'entretiens ont été réalisés : entretien ante et post séance.

\subsubsection{L'entretien ante séance}

C'est un entretien semi directif dont les items abordent les aspects relatifs aux objets d'apprentissage de la séance. Il est focalisé sur des points comme la situation de la séance dans le cycle, le choix des objectifs de la séance, le choix des activités ou tâches pour développer tel ou tel aspect du thème d'étude, les difficultés éventuelles que les élèves pourraient rencontrer dans l'exécution des activités d'apprentissage.

\subsubsection{L'entretien post séance}

De type semi directif, il est réalisé juste après l'enregistrement de la séance avec l'enseignant. L'objectif est de recueillir les traces à propos des activités didactiques telles que ressenties par le professeur, afin d'éviter d'attribuer à l'enseignant des points de vue de l'observateur. Il faut noter que dans ce protocole, les élèves n'ont pas été interrogés, parce que nos questions de recherche sont principalement centrées sur les contraintes et les possibles de la mise en œuvre des NPE-EPS du côté du sous-système enseignant, relativement au fonctionnement du système didactique observé.

\subsection{Les observations}

Trois séances ont été observées dans une classe de cinquième de 63 élèves lors d'un cycle de basket-ball. Elles se situent au début des apprentissages dans le cycle. Les observations ont pris en compte les trois phases d'une situation d'apprentissage ("introduction", " réalisation ", " retour et projection ») conformément aux prescriptions des NPE-EPS. La phase d'introduction est caractérisée par la mise en œuvre d'une «situation de départ ». 
Cette situation de départ vise selon de l'APC à contextualiser l'apprentissage dans le cadre des aspects de la vie sociale. Dans le cadre de cette étude, chaque séance filmée est considérée comme une unité d'analyse. Nous disposons donc de trois unités d'analyse.

\subsection{L'analyse des données} réalisée. La condensation des données vidéo et audio a été effectuée à l'aide d'un synopsis qui met en relation les échanges verbaux en classe, l'action des élèves, les consignes et actions de l'enseignant (Amade-Escot, 2003). Nous avons utilisé un synopsis dit « expansé ». Ce synopsis propose un niveau de condensation intermédiaire entre celui proposé par Leutenegger (2009) qui porte sur les données au niveau mésoscopique d'une seule séance et celui proposé par Schneuwly, Dolz et Ronveau (2006) qui, pour sa part, relève d'un niveau macroscopique de transcription d'un ensemble de séances, afin de rendre compte de l'évolution du savoir enseigné sur une échelle temporelle longue (6 mois à 1 an). L'intérêt de ce type de synopsis (dit «expansé ») réside dans le fait qu'il permet d'accéder aux consignes données par l'enseignant et aux conduites typiques des élèves dans les différentes tâches d'apprentissage. Il met en relation les transcriptions verbales (le contenu des tours de parole du professeur et des élèves), les actions des élèves, au regard des commentaires interprétatifs du chercheur en lien avec les questions de recherche. Ce synopsis expansé permet d'accéder à la dynamique de mise en œuvre des NPE-EPS dans la mesure où il rend compte du curriculum en actes sur une échelle de temps assez longue (ici trois séances). L'analyse qualitative de ces synopsis se fonde sur un système de protocole mettant en correspondances plusieurs corpus : croisement entre les données d'observation et les éléments de discours des différents acteurs. Ce processus est appelé "triangulation» dans les manuels de recherche qualitative (Vander Maren, 1996). Le niveau de triangulation concerne la ternarité des systèmes didactiques. Il s'agit de combiner les traces : analyse a priori des savoirs mis en jeu dans les tâches ; intentions didactiques du professeur et leur évolution, les actions des élèves en lien avec les savoirs enseignés.

\section{Résultats}

Abordons les résultats relatifs à la mise en évidence de la succession des objets mis à l'étude au cours des trois séances de basket-ball observées. Il s'agit donc de la construction de référence au fil des trois séances, les liens entre les constats effectués et l'épistémologie de ce conseiller-pédagogique au regard des préconisations officielles des NPE-EPS et des caractéristiques du système didactique observé.

\subsection{La construction de référence au fil des trois séances}

21

Elle concerne les savoirs en jeu et la démarche de construction de ces savoirs, synthétisés dans le tableau ci-après qui résument les trois observations. 
Tableau 2. Synthèse des observations : conception du basket-ball enseigné et savoirs mis à l'étude au fil des 3 séances.

\begin{tabular}{|c|c|c|c|}
\hline & Séance 1 & Séance 2 & Séance 3 \\
\hline Thème de séance & $\begin{array}{l}\text { Jouer au basket- } \\
\text { ball dans sa forme } \\
\text { globale }\end{array}$ & $\begin{array}{l}\text { - Apprentissage de la } \\
\text { passe-réception } \\
\text { - Éclatement }\end{array}$ & $\begin{array}{l}\text { - Apprentissage du } \\
\text { dribble et du tir au } \\
\text { basket-ball }\end{array}$ \\
\hline But de séance & $\begin{array}{l}-\quad \text { Identification } \\
\text { des étapes } \\
\text { - } \quad \text { Règlement du } \\
\text { basket-ball }\end{array}$ & \begin{tabular}{lr}
\multicolumn{4}{|c}{ Progression en passe } \\
vers le r panier \\
adverse \\
- Identifier r les \\
principes d'une bonne \\
passe
\end{tabular} & $\begin{array}{l}\text { - Apprendre à dribbler } \\
\text { - Apprendre à tirer au } \\
\text { panier } \\
\text { - Apprendre à faire le } \\
\text { pivot }\end{array}$ \\
\hline $\begin{array}{l}\text { Tâches proposées } \\
\text { Approche de } \\
\text { l'enseignement du basket- } \\
\text { ball (Brau Antony, 2003) }\end{array}$ & $\begin{array}{l}-\quad 3 \quad \text { tâches } \\
\text { relevant d'une } \\
\text { approche } \\
\text { techniciste } \\
\text { - } 1 \text { tâche relevant } \\
\text { de l'approche } \\
\text { structurale }\end{array}$ & $\begin{array}{lr}-\quad 5 \text { tâches } & \text { relevant } \\
\text { d'une } & \text { approche } \\
\text { techniciste } & \end{array}$ & $\begin{array}{l}\text { - } 3 \text { tâches relevant } \\
\text { d'une approche } \\
\text { techniciste } \\
\text { - } 1 \text { tâche relevant de } \\
\text { l'approche structurale }\end{array}$ \\
\hline $\begin{array}{l}\begin{array}{l}\text { Savoirs du basket-ball } \\
\text { réellement enseignés } \\
\text { (Marsenach, 1991) }\end{array} \\
\text { (M) }\end{array}$ & $\begin{array}{l}\text { - Enonciation des } \\
\text { savoirs } \\
\text { élémentarisés à } \\
\text { construire } \\
\text { - Règle de jeu }\end{array}$ & $\begin{array}{l}\text { - Elémentarisation des } \\
\text { savoirs techniques de } \\
\text { passe et de réception } \\
\text { - Principes techniques } \\
\text { d'une passe } \\
\text { - Placement sur } \\
\text { l'espace de jeu } \\
\text { - Règles de jeu }\end{array}$ & $\begin{array}{l}\text { - Elémentarisation des } \\
\text { savoirs techniques : } \\
\text { dribble, tir, arrêt et } \\
\text { pivot } \\
\text { - Intégration des } \\
\text { savoirs techniques } \\
\text { dans le jeu }\end{array}$ \\
\hline
\end{tabular}

\subsubsection{Au niveau des objets de savoirs}

L'ensemble des objets mis à l'étude pendant les trois séances de basket-ball observées est identifiable, selon les synopsis analysés, à travers des tâches, des moments didactiques et des temps didactiques. Ces tâches, moments et temps didactiques ont été mis en évidence à travers trois objets techniques: la passe, le dribble et le tir (Cf. tableau 2 récapitulatif des objets réellement enseignés). La presque quasi-totalité des tâches proposées résultent d'une approche techniciste du basket-ball (Brau-Antony, 2003). Quant aux savoirs réellement enseignés, ils sont constitués de gestes techniques très élémentarisés : la passe-réception, le dribble et le tir auxquels s'ajoute le pivot en tant que modèle gestuel à reproduire (Marsenach, 1991). Tous ces éléments ont été abordés au fil des trois séances dans des situations fortement décontextualisées. L'analyse a montré que les conceptions de cet enseignant ont eu une influence sur ses choix didactiques comme l'a pointé Brau Antony (2003). Au niveau de la conception de l'enseignement du basket-ball, cet enseignant propose des tâches élémentarisées relevant d'une conception techniciste: 
ainsi les tâches d'apprentissage proposées aux élèves sont fortement décontextualisées, épurées, présentées sous forme de gammes d'exercices "à vide", c'est-à-dire sans opposition, ni même parfois sans cible à atteindre (Bouthier, 1986). L'enseignant a certes proposé une variété de tâches concernant surtout l'apprentissage de la passe, mais ces dernières n'ont nullement contribué à faire progresser de façon significative les élèves du point de vue de la maîtrise du jeu. La tâche de montée collective du ballon reste isolée de la dynamique du jeu. Nous n'avons jamais observé de mises en œuvre basées sur une approche dialectique du basket-ball avec la présence d'opposition, alors que les recherches ont montré que l'alternance de situations en plan collectif total ou réduit sont susceptibles de permettre le développement de compétences spécifiques au jeu collectif (Brau Antony, 2001 ; Bouthier, 1986). Ce n'est qu'à la dernière séance que le temps de jeu a été plus conséquent. Face aux échecs des élèves, les régulations effectuées ont consisté soit à un réaménagement du dispositif, soit à une démonstration du geste à reproduire de l'enseignant.

\subsubsection{Au niveau de la démarche d'apprentissage}

Conformément aux principes de l'APC et aux contenus préconisés par les NPE-EPS, la démarche d'enseignement et d'apprentissage constitue un objet d'apprentissage (Agbodjogbé, 2013). Or, l'analyse des trois séances a montré qu'en dehors des quatre capacités de la démarche d'enseignement et d'apprentissage préconisées formellement par les textes officiels (expression, analyse, planification, exploration: voir tableau 1, dernière colonne), les objets mis à l'étude relèvent d'une élémentarisation de savoirs techniques sans lien avec leurs conditions d'utilisation dans un jeu d'opposition, ainsi que de quelques éléments du code de jeu du basket-ball surtout lors de la séance 1. Nous n'avons observé aucune tâche d'opposition, à l'exception de quelques courtes minutes de jeu développé en fin de séance. On est donc loin de la compétence pourtant énoncée par les NPE-EPS : « Pratiquer collectivement l'activité physique ».

L'analyse des objets réellement enseignés pose deux problèmes: celui des contenus d'enseignement et celui de la transposition didactique. Cet enseignant, à défaut d'amener les élèves à développer des compétences par l'intermédiaire d'une démarche d'enseignement/apprentissage prescrite par les textes officiels, met en place une approche décontextualisée de l'enseignement du basket-ball très peu en prise avec les problématiques de construction de compétences qui soient une image de la pratique sociale de référence (Martinand, 1994). La transposition des objets d'apprentissage opérée par ce professeur semble peu prendre en compte l'épistémologie de la discipline.

\subsection{Singularité du système didactique observé}

En référence, à l'inscription théorique de cette étude se dégagent certaines caractéristiques qui révèlent les modalités d'implémentation des NPE-EPS par cet enseignant.

\subsubsection{Une dévolution difficile}

$\mathrm{Au}$ fil des séances, nous avons constaté des ruptures du contrat didactique qui provoquent des glissements vers des effets topaze lors desquels l'enseignant énonce le savoir attendu. Les stratégies contractuelles de l'enseignant sont basées sur des 
questions-réponses-évaluation soutenues par des pratiques ostensives. Il lui est donc difficile dans cette perspective de transférer aux élèves la responsabilité de la construction des savoirs telle que préconisée par les NPE-EPS selon les principes socioconstructivistes de l'APC. Les tâches proposées aux élèves sont par ailleurs de faible épaisseur technologique au sens de Chevallard (2002). Les formes de direction d'étude contribuent à affaiblir leur densité épistémique.

La logique d'ostension qui caractérise la pratique de cet enseignant a pour objectif premier de montrer aux élèves l'image de référence (démonstrations à reproduire). Les régulations se présentent sous forme d'injonctions permanentes auprès des élèves lors de l'accomplissement des tâches motrices. Elles donnent lieu à de longues explications, caractéristiques d'un enseignement transmissif selon des contrats didactiques d'émission et de conditionnement (Brousseau, 1996).

\subsubsection{Une posture topogénétique en surplomb associée à une chronogenèse quasi immobile}

Pendant la construction de référence, professeur et élèves occupent des positions dissymétriques. L'analyse des trois séances a montré la prépondérance d'une posture topogénétiques haute de la part de ce professeur, ce qui peut sembler étonnant au regard de la démarche "socioconstructiviste " de l'APC à laquelle il déclare être attaché (entretien). En fait, nous avons observé des formes alternées de topogenèse avec des moments où une très grande latitude d'action est laissée aux élèves (dans les jeux dirigés) justifiés dans les entretiens en référence à la construction des savoirs par les élèves euxmêmes et des moments (les plus nombreux) où les explications et les démonstrations qui précèdent ou succèdent l'exécution des tâches sont conduites de façon très directive, selon un topos très surplombant, en contradiction avec les discours tenus. Ce constat peut apparaitre paradoxal compte-tenu du statut de conseiller-pédagogique de cet enseignant (membre de la noosphère). La plupart des tentatives de transfert de responsabilités ont été observées lors des échanges organisés en "plénière " (moments préconisés par le NPE-EPS où la classe est réunie pour échanger sur ce qu'elle vient de réaliser). L'analyse des interactions verbales professeur-élèves met en évidence une conception de la dévolution telle que pensée par les NPE-EPS, en relation avec des présupposés socioconstructivistes assez radicaux, tirés de l'APC. Tout se passe comme si l'expérience du jeu suffisait à permettre à l'élève de « construire les savoirs » et d'en rendre compte lors des échanges. L'idée que l'expérience des élèves puisse être guidée par observations ou des consignes précises en lien avec une analyse de la logique interne du jeu de basketball n'est jamais évoquée par cet enseignant. Or, l'impossibilité avérée au fil des séances d'obtenir de telles avancées, malgré quelques transferts sporadiques constatés, ne peuvent déboucher que sur une reprise en main de l'enseignant sous forme d'effets Topaze ou Jourdain, en surplomb, marquant l'immobilité de l'avancée de savoir. Cette posture du point de vue des NPE-EPS pose le problème du rôle assigné dans les textes à l'enseignant (celui d'un «accompagnateur »). Les résultats de cette étude, qui rendent compte du curriculum en actes tel qu'il se joue dans ce système didactique singulier, en pointent toutes les difficultés.

29 Les différents constats établis confortés par d'autres études de cas (Agbodjogbé, 2013; Amade-Escot \& Agbodjogbé, 2013) suggèrent que pour endosser un rôle « d'accompagnateur des apprentissages des élèves » les professeurs puissent disposer des 
outils didactiques pouvant leur permettre d'analyser les conduites des élèves. Ils invitent à mettre en débat la question des fondements épistémologiques de l'APC.

\section{Discussion conclusive}

30 Au Bénin, l'implémentation des nouveaux programmes par compétences a mis en évidence de nombreuses difficultés d'opérationnalisation liées entre autre à la démarche d'enseignement et d'apprentissage préconisée par les textes officiels, mais aussi et surtout au choix des objets d'enseignement, des savoirs y afférant et des modalités de leur construction. Au-delà des controverses politiques, épistémologiques, théoriques et pratiques mises en évidence par la littérature sur les orientations curriculaires contemporaines, la question de la référence à construire, telle que préconisée par les NPE-EPS au Bénin, reste encore problématique surtout dans les sports collectifs. L'étude de cas dont nous venons de rendre compte au niveau du basket-ball en est un exemple. Elle visait à documenter les questions de recherche suivantes: à quelles pratiques scolaires de référence sont confrontés les élèves en basket-ball ? Comment sont mis en œuvre ces contenus d'enseignement au basket-ball au regard de la nouvelle matrice disciplinaire introduite par la réforme selon l'APC?

Le protocole de Leutenegger (2009) nous a permis de réaliser des observations didactiques ordinaires. Nous nous sommes intéressés aux interactions développées par les protagonistes de l'action didactique au cours de trois séances. De l'analyse des résultats obtenus, il ressort que la pratique de cet enseignant d'EPS, par ailleurs conseillerpédagogique impliqué dans le suivi de la réforme, se caractérise par une mise en œuvre assez respectueuse des attendus formels des NPE-EPS tant au niveau des contenus que des démarches et des formes de travail utilisées. Les questions relatives aux objets d'apprentissage sont soit posées avant le démarrage, soit lors de la discussion avec le groupe-classe. Cet enseignant reste ainsi assujetti aux préconisations officielles, ce qui l'amène moins à traiter des difficultés rencontrées par ses élèves en termes d'apprentissage du jeu collectif qu'à s'attacher à une forme de socioconstructivisme naïf, mis en œuvre dans certains moments de la séance: lors des échanges en plénière. En revanche, dans les autres moments de la séance, lorsque les élèves sont confrontés à des tâches appelées dans les textes officiels "activités de réalisation" ou "activités d'exécution », on a pu observer un recours fréquent à la démonstration, une faible densité en savoirs des milieux didactiques proposés aux élèves, des régulations caractérisées par une très grande centration sur des modèles gestuels décontextualisés. Ces constats éclairent un autre aspect de l'épistémologie pratique de cet enseignant et témoignent d'une démarche assez transmissive. Les modalités formelles d'interaction laissent penser que pour cet enseignant, il suffit de poser des questions aux élèves, de les faire échanger pour que leurs réponses produisent des effets dans l'action au niveau du jeu. Au final, l'implémentation des NPE-EPS par cet enseignant semble pouvoir être résumée selon deux dimensions : la faiblesse épistémique des contenus d'enseignement associée à un assujettissement aux principes généraux de l'APC. Relativement à la faiblesse épistémique, il ressort que les trois séances analysées sont caractérisées par un traitement en surface des contenus abordés, selon des modalités transpositives très technicistes révélées par la structuration des tâches proposées aux élèves. En ce qui concerne l'assujettissement de cet enseignant à une forme naïve de socioconstructivisme, il nous semble pouvoir être expliqué par les institutions traversées (Chevallard, 1996), 
notamment par son statut de conseiller-pédagogique lors de la mise en place des NPE-EPS qu'il a par ailleurs contribué à produire. En tant que membre de la noosphère, il ne peut se saisir des contradictions résultant des choix curriculaires effectués en EPS et n'a d'autre alternative que de donner à voir dans certains moments de ses séances «les bonnes manières de faire construire le savoir aux élèves ", alors que dans d'autres, il mobilise les usages professionnels sédimentés d'une pédagogie de maîtrise.

Ces constats qui recoupent ceux établis par une autre étude portant sur l'enseignement de la course de haies en quatrième (Amade-Escot \& Agbodjogbé, 2013) posent plus largement la question des effets des réformes curriculaires sur les pratiques didactiques des enseignants. Ils interrogent les processus de reconfiguration curriculaire tels que soutenus par les organismes supra nationaux et les effets des choix pédagogiques et didactiques opérés sur le curriculum mis en œuvre dans le quotidien des classes.

\section{BIBLIOGRAPHIE}

Agbodjogbé, B. (2013). L'implémentation des nouveaux programmes par compétences au Bénin: des textes officiels aux pratiques d'enseignement. Analyses didactiques en Éducation Physique et Sportive et en Sciences de la Vie et de la Terre en classe de 5ème. Thèse de doctorat de Sciences de l'Éducation, non publiée, Université de Toulouse, Toulouse-Le-Mirail.

Agbodjogbé, B., Amade-Escot, C., \& Attiklèmè, K. (2013). La réforme des curriculums au Bénin : le point de vue des acteurs en EPS et en SVT. Education et Socialisation - Les Cahiers du CERFEE, 34. En ligne http://edso.revues.org/419

Amade-Escot, C. \& Agbodjogbé, B. (2013). Les apories de l'enseignement de l'EPS par « compétences » au Bénin : analyse didactique d'une séance de haies conduite par un conseiller pédagogique. Communication au 3ème Colloque de l'ARCD, Marseille, 9-12 janvier.

Agbodjogbé, B. (2007). Problématique de mise en œuvre de la démarche d'enseignement apprentissage évaluation en EPS selon les programmes par compétences au Bénin. Mémoire de Master 2, non publié, INJEPS, Université d'Abomey-Calavi, Porto-Novo.

Amade-Escot, C. (2007). Les savoirs au cœur du didactique. In C. Amade-Escot, (Coord), Le didactique, (pp. 11-30). Paris : édition Revue EPS, Collection « Pour l'action ».

Amade-Escot, C. (2003). La gestion interactive du contrat didactique en volleyball : agencement des milieux et régulation du professeur. In C. Amade-Escot (Ed.), Didactique de l'éducation Physique, Etat des recherches (pp. 233-253). Edition Revue EPS, Paris.

Attiklèmè, K. (2009). Les contenus du nouveau programme d'EPS définis en termes compétences au Bénin : une problématique de l'intervention de l'enseignant du secondaire. Revue STAPS, 86, 93-108.

Bouthier, D. (1986). Comparaison expérimentale des effets des différents modèles didactiques des sports collectifs. In SNEP, EPS : Contenu et didactique (pp. 85-89). Paris : SNEP. 
Brau-Antony, S. (2001). Les conceptions des enseignants d'éducation physique et sportive sur l'enseignement et l'évaluation des jeux sportifs et collectifs : résultats d'une enquête. Revue STAPS , 56, 93-108.

Brau-Antony, S. (2003). Caractéristiques des situations didactiques proposées par des enseignants d'EPS dans le cadre de l'enseignement du volleyball. In C. Amade-Escot (Ed.), Didactique de l'éducation physique et sportive. Etat des Recherches pp. 169-192). Paris : Revue EPS, Collection Recherche et Formation.

Brousseau, G. (1996). L'enseignant dans la théorie des situations didactiques. In R. Noirfalis et M.J Perrin-Glorian (Eds), Actes de la VIIIème école d'été de didactique des mathématiques (pp. 3-46). Clermont Ferrand.

Crahay, M., Audigier, F., \& Dolz, J. (2006). En quoi les curriculums peuvent-ils être objets d'investigation scientifique ? In A. Audigier, M. Crahay, J. Dolz. Curriculum, enseignement et pilote (pp. 7-37). Bruxelles : Edition De Boeck.

Chevallard, Y. (1991). La transposition didactique. Du savoir savant au savoir enseigné. Grenoble : La Pensée sauvage. $2^{\mathrm{e}}$ édition augmentée.

Chevallard, Y. (1997). Familière et problématique, la figure du professeur. Recherches en Didactique des Mathématiques, 17(3), 17-54.

Chevallard, Y. (2002). Approche anthropologique du rapport au savoir et didactique des mathématiques. In Actes des 3èmejournées d'études Franco-Québécoises des didactiques. Didactique et Rapports aux savoirs (pp. 182-197). Paris V, la Sorbonne : Laboratoire Education et Apprentissage (E. D. A).

Cros, F., Dembélé, M. De Ketele, J.-M., Develay, M., Gauthier, R.-F., Ghriss, N., Lenoir, Y., Murayi, A., Suchaut, B., Tehio, V. (2009). Étude sur les réformes curriculaires par l'approche par compétences en Afrique. Sèvres : Centre international d'études pédagogiques. En ligne https://depot.erudit.org/ id/003225dd

Jonnaert, P. (2002). Compétence et Socioconstructivisme un cadre théorique. Bruxelles : De Boeck ; Perspectives Education et Formation. .

Jonnaert, P., Ettayebi, M., \& Defise, R. (2009). Curriculum et compétences un cadre opérationnel. Bruxelles : De Boeck Université, Perspective en éducation et formation.

Leutenegger, F. (2009). Le temps d'instruire. Approche clinique et expérimentale du didactique ordinaire en mathématique. Berne : Peter Lang.

Martinand, J.L. (1994). Didactique des sciences et formation des enseignants. Notes d'actualité. Les sciences de l'éducation pour l'ère nouvelle, 2, 23-29.

Marsenach, J. (1991). Education Physique et Sportive. Quel enseignement ? Paris : INRP.

OCDE (2008). Regards sur l'éducation. Les indicateurs de l'OCDE. PARIS : OCDE.

Roegier, X. (2008). L'approche par compétence en Afrique francophone : quelques tendances. Bureau international de l'UNESCO: IBE working Paper on Curriculum Issues, 7, 23-30

Schneuwly, B., Dolz, J., \& Ronveau, C. (2006). Le synopsis : un outil pour analyser les objets enseignés. In M. J. Perrin-Glorian et Y. Reuter (Eds), Les méthodes de recherche en didactiques (pp. 175-189). Villeneuve d'Asq : Edition du Septentrion.

Ogueboule, B. (2008). Les effets emblématiques du nouveau programme d'EPS au Bénin :le cas de la SA1 dans les classes de $6^{\text {ème }}$ des établissements secondaires de l'Ouémé. Mémoire de Master, non publié, Université d’Abomey-Calavi, INJEPS, Porto-Novo, République du Bénin. 
UNESCO(2000). Cadre d'action de Dakar. L'éducation pour tous, tenir nos engagements collectifs avec les six cadres d'action régionaux, adoptés par le Forum mondial sur l'éducation, Dakar, Sénégal, 2 6-28 avril.

Schubauer-Leoni M. L. \& Leutenegger, F. (2002). Expliquer et comprendre dans une approche clinique expérimentale du didactique ordinaire. In F. Leutenegger et M. Saada-Robert (Eds), Expliquer et comprendre en sciences de l'éducation (pp. 227-251). Bruxelles, De Boeck, Raisons éducatives.

Schubauer-Leoni, M-L. et Leutenegger, F. (2005). Une relecture des phénomènes transpositifs à la lumière de la didactique comparée. Revue Suisse des sciences de l'éducation, 77, 407-429.

Sensevy, G. (2007). Des catégories pour décrire et comprendre l'action didactique. In G. Sensevy et A. Mercier (Eds). Agir ensemble l'action didactique conjointe du professeur et des élèves (pp. 13-49). Rennes : Presse Universitaire de Rennes.

Van Der Maren, (1996). Méthodes de recherche pour l'éducation. Bruxelles : De Boeck Université.

Zomahoun, C. (2008). Le système éducatif béninois de la période coloniale à nos jours : une approche comparée des contenus enseignés en EPS au secondaire. Mémoire de Maîtrise en STAPS ; Université d'Abomey-Calavi, INJEPS, Porto-Novo, République du Bénin.

\section{RÉSUMÉS}

Le vaste mouvement curriculaire dans lequel la plupart des pays se sont engagés depuis plus de deux décennies a permis à plusieurs d'entre eux, de réorienter leur système éducatif et de formation vers l'approche par les compétences (APC). Le Bénin, à l'instar de certains pays de l'Afrique subsaharienne n'est pas resté en marge de cette « révolution curriculaire ». Il s'est donc engagé dans une refonte de ces programmes dans toutes les disciplines notamment en EPS. Leur mise en œuvre a très tôt soulevé de nombreuses controverses aux plans sociopolitique, épistémologique pédagogique et surtout didactique. La présente étude propose l'analyse de la pratique enseignante d'un conseiller-pédagogique lors d'un cycle de basket-ball. Elle rend compte, sous couvert de la théorie de l'action conjointe en didactique de la manière dont cet enseignant implémente les nouveaux programmes en EPS dans un cycle de basket-ball. L'analyse de trois séances conduites met en évidence une démarche transmissive qui traduit des usages professionnels relevant des principes de la pédagogie par objectifs, selon une posture en surplomb. Les objets enseignés, les modalités de travail instaurées, s'ils sont conformes à ceux préconisés par les textes officiels se caractérisent par la faiblesse épistémique des contenus abordés. Du point de vue du curriculum en acte, ce conseiller pédagogique valorise les dimensions de surface de la démarche préconisée au détriment des contenus d'enseignement dont la maîtrise reste aussi problématique.

The broad curricula reform in which most of countries have been involved over two decades has enable many of them to reorient their educational and training system to the so-called "competence based curriculum". The Republic of Benin, like some other countries in sub-Saharan Africa does not remain on the side-lines of this "curriculum revolution". The reform was engaged in all disciplines including Physical Education which adopted new programs based on the competence approach. However, the reform has raised many controversies (i.e. socio-political, educational, epistemological and didactical). This study analyses the teaching of a basket-ball unit at a middle school level. It examine against the background of the joint action theory in didactics (Sensevy, 2007) how a teacher implements the PE new programs during three lessons. The teacher, who is involved as an academic advisor in the reform, used a very traditional 
approach that reflects his professional habits rooted an objectives approach of mastery learning, associated with a strong overhang posture. The taught objects, the classroom management used by this teacher are in compliance with those recommended by the official texts, but have for consequences to value the surface dimensions of the competence based approach at the expense of the complexity of knowledge content really learnt which the epistemological mastery by this academic advisor remains also critical.

\section{INDEX}

Keywords : competence approach, physical education, joint action in didactics, didactic transposition

Mots-clés : approche par compétence, éducation physique et sportive, action conjointe en didactique, transposition didactique

\section{AUTEURS}

\section{BASILE AGBODJOGBÉ}

Assistant, Université d'Abomey- Calavi, INJEPS, République du Benin, Chercheur associé à l'UMREFTS, Université de Toulouse 2 - Jean Jaurès

\section{KOSSIVI ATTIKLÉMÉ}

Maître de conférences, Université d'Abomey-Calavi, Laboratoire de Didactiques des Disciplines, INJEPS, République du Benin

\section{CARLOS ATOUN}

Doctorant, Université d'Abomey-Calavi, INJEPS, République du Benin 